\title{
GERENCIAMENTO DE NOVAS TECNOLOGIAS EM CENTRO CIRÚRGICO PELAS ENFERMEIRAS NOS HOSPITAIS DE FEIRA DE SANTANA - BA
}

Marluce Alves Nunes Oliveira*

\section{Resumo}

Trata-se de estudo quantitativo exploratório descritivo sobre o nível de adequação ao domínio das novas tecnologias (NADNT) das enfermeiras no gerenciamento de centro cirúrgico em hospitais de Feira de Santana BA - Brasil, 2002. Teve como objetivos: avaliar o NADNT das enfermeiras no gerenciamento de centro cirúrgico (CC); identificar e descrever os fatores que interferem neste nível, bem como propor ações para o desenvolvimento deste nível no gerenciamento de CC. Amostra constituída por 33 enfermeiras com experiência de CC. Utlizou-se questionário para a coleta de dados. Conclui-se que o NADNT foi insatisfatório por $54,5 \%$ dos sujeitos do estudo. Comprovou-se estatisticamente que a capacidade do hospital e a carga horária são fatores que interferem no domínio das novas tecnologias em CC.

Descritores: centros cirúrgicos; novas tecnologias/centros cirúrgicos; gerenciamento/ enfermagem em centros cirúrgicos

\begin{abstract}
It is a quantitative, exploratory, descriptive study about the level of adequacy of the command of new technologies (NADNT) by nurses in the management of a surgery center in hospitals located in Feira de Santana-BA, Brazil, in 2002. It aimed at: assessing the NADNT of nurses in the management of a surgery center; identifying and describing the factors that interfere with this level; and proposing actions for the development of this level in the management of surgery centers. Sample made up of 33 nurses with experience in surgery centers. A questionnaire was used for data collection. It has been concluded that the NADNT was unsatisfactory for $54.5 \%$ of the study subjects. It has been statistically proved that the capacity of the hospital and the working load are factors that interfere with the command of new technologies in surgery centers.
\end{abstract}

Descriptors: surgery centers; new technologies/surgery centers; management/ nursing at surgery centers

Title: Management of new technologies in a surgery center by nurses in hospitals located in Feira de Santana - BA

\section{Resumen}

Se trata de un estudio cuantitativo explorador descriptivo sobre el nivel de adaptación del dominio de las nuevas tecnologías (nadnt) de las enfermeras en la administración del centro quirúrgico en hospitales de Feira de SantanaBA - Brasil, 2002. Tuvo como objetivos: evaluar el nadnt de las enfermeras y la administración del centro quirúrgico (cc) en los hospitales investigados, identificar y describir los factores que interfieren en este nivel, así como proponer acciones para el desarrollo de este nivel en la administración del (cc). La muestra se constituyó de 33 enfermeras con experiencia en (cc). Se utilizó cuestionario para la información de datos. Conclusiones: el dominio das las nuevas tecnologías fue considerado insatisfactorio por $54,5 \%$ de los sujetos del estudio. Estadísticamente, se comprobó que la capacidad del hospital y la carga horaria son factores que interfieren en el dominio de las nuevas tecnologías en cc.

Descriptores: centros quirúrgicos, nuevas tecnologías/centros quirúrgicos; administración/ enfermería en centros quirúrgicos.

Título: Administración de nuevas tecnologías en centro quirúrgico por las enfermeras en los hospitales de Feira de Santana-BA

\section{Introdução}

A acepção enfermeira gerente de centro cirúrgico é compreendida como a profissional responsável pelos serviços em que o cuidado perioperatório é ministrado ao paciente, independente do tipo de hospital em que atua: filantrópico, privado ou público.

Dentre as diversas unidades da estrutura hospitalar, destaca-se o centro cirúrgico, uma unidade que contém um conjunto de elementos destinados às atividades cirúrgicas, constituindo assim, o setor que tem como prioridade prestar assistência de qualidade ao paciente, desde o recebimento no pré-operatório imediato até a recuperação pós-anestesica. Sabe-se que é fundamental obter-se condições adequadas no ato anestésico-cirúrgico, tanto para segurança física e psicológica do paciente quanto da equipe cirúrgica.

Ultimamente, presencia-se, no cotidiano da unidade de centro cirúrgico, um aumento exponencial de complexidade tecnológica, científica e de relações humanas, gerando, dessa forma, um novo perfil para a gerente dessa unidade ${ }^{(1)}$.

É importante que a enfermeira de centro cirúrgico compreenda que a tecnologia pode contribuir no fortalecimento da capacidade de inovar a assistência ao paciente no perioperatório. As exigências do mundo contemporâneo observado no cotidiano, à vista da utilização da ciência e da tecnologia, repercutem cada dia mais na vida das pessoas, mostram, com grande ênfase, que a sociedade deve se organizar para adaptar-se aos novos tempos. Impõe-se, portanto, a necessidade de atualização das enfermeiras de centro cirúrgico para tornarem-se profissionais eficazes e sintonizados com as novas exigências do mercado de trabalho. Considerando o valor das novas tecnologias (biomédica, comunicação e informação) na assistência hospitalar, é necessário que os profissionais de saúde tenham a clareza que esta assistência deve ser pautada pelo respeito a vida humana e pela observância dos princípios ético-morais no convívio entre profissionais e pacientes.

O presente estudo foi suscitado ao ingressar na Universidade Estadual de Feira de Santana como docente da disciplina Enfermagem Cirúrgica, e atualmente, na disciplina Enfermagem na Saúde do Adulto e Idoso II, do Curso de Enfermagem, havia sempre o questionamento sobre a atuação das enfermeiras em centro cirúrgico, no que diz respeito à assistência ao paciente no perioperatório. Daí, a motivação para realizar-lo, a partir da vivência no campo da prática com os estudantes do $7^{\circ}$ semestre do Curso de Enfermagem, nos centros cirúrgicos dos hospitais públicos e privados do Município de Feira de Santana.

Observou-se a ausência da enfermeira no início do intraoperatório, momento em que a presença desse profissional é muito importante, a fim de transmitir confiança, segurança e proporcionar uma assistência de qualidade ao paciente, colaborando assim, com toda a equipe cirúrgica. Diante dessa realidade, o questionamento: Por que a enfermeira durante o intraoperatório se ausenta da sala de operação? Como a enfermeira gerencia as novas tecnologias? Como a tecnologia está inserida no processo de trabalho da enfermeira? O nível de adequação ao domínio das novas tecnologias é satisfatório ou insatisfatório no gerenciamento da enfermeira em centro cirúrgico?

\footnotetext{
* Enfermeira. Mestre em Engenharia de Produção na área de Mídia e Conhecimento. Professora Assistente do Curso de Graduação em Enfermagem da Disciplina Enfermagem na Saúde do Adulto e Idoso II do Departamento de Saúde da Universidade Estadual de Feira de Santana.

E-mail do autor: milicialves@yahoo.com.br
} 
Neste estudo, o padrão de qualidade estabelecido é nível de adequação ao domínio das novas tecnologias satisfatório (NADNTS) e nível de adequação ao domínio de novas tecnologias insatisfatório (NADNTI).

O NADNTS deve ser compreendido como o conhecimento e habilidade da enfermeira em efetuar a assistência por meio dos recursos tecnológicos (equipamentos e materiais) de máxima efetividade em tratamento dispensado ao paciente, com eficácia e segurança, a fim de contribuir na assistência de enfermagem com qualidade. Quanto à tecnologia da informação e comunicação, é considerada satisfatória, quando essa é exercida no gerenciamento e na administração dos serviços e como suporte à prática assistencial, considera-se o NADNTI, quando essas atividades são realizadas em forma de processo eventualmente ou não.

O presente estudo teve como objetivo geral: avaliar o NADNT por parte das enfermeiras no gerenciamento em centro cirúrgico de hospitais, e como objetivos específicos: Identificar e descrever os fatores que interferem no NADNT, bem como propor ações para o desenvolvimento no NADNT pelas enfermeiras no gerenciamento em centro cirúrgico de hospitais.

\section{Marco Teórico}

No trabalho da enfermeira de centro cirúrgico deve-se procurar alcançar a qualidade através das novas tendências do mundo contemporâneo, a fim de buscar o equilíbrio entre eficácia e eficiência.

Sabe-se que o centro cirúrgico é uma das unidades mais complexas do hospital, pela sua especificidade, pelo estresse e a grande probabilidade de expor o paciente a riscos da saúde, ao serem submetidos a intervenções cirúrgicas.

Portanto, é responsabilidade da enfermeira do centro cirúrgico proporcionar estrutura física, recursos humanos e materiais para que o ato anestésico-cirúrgico se realize em condições ideais, visando assistência integral, o ensino e a pesquisa.

Enfim, é da responsabilidade da enfermeira perioperatória gerenciar a unidade com responsabilidade e competência, estabelecer condutas éticas a toda a equipe do centro cirúrgico, manter um ambiente seguro e educar o paciente a respeito de sua doença, tratamento, promoção a saúde e autocuidado, assim promover o cuidado ao paciente cirúrgico com qualidade(2). Assim, "o trabalho da enfermagem compreende quatro processos: cuidar/assistir, administrar/ gerenciar, ensinar/educar e investigar/pesquisar"(3:68).

Será dada ênfase ao processo gerenciar/administrar da enfermagem. Nessa maneira de gerenciar, a enfermeira de centro cirúrgico coordena e lidera atividades assistenciais, atividades administrativas e atividades tecnológicas.

A tecnologia, em todas as áreas, evolui muito rapidamente, é necessário, portanto, que a enfermeira que gerencia o centro cirúrgico, desenvolva novas habilidades e novos conhecimentos referentes à tecnologia para que ofereça ao paciente uma assistência de qualidade e com vantagens competitivas.

Neste sentido, a rápida obsolescência do conhecimento causada pelas contínuas mudanças tecnológicas e mercadológicas impõe a necessidade de transformar o aprendizado em uma prática constante ${ }^{(4)}$.

O termo tecnologia provém de técnica que, de acordo com o vocabulário latino, significa arte ou habilidade, então, depreende-se que a tecnologia é uma atividade que está ligada à prática(5)

Dessa forma, aplicar o conhecimento da tecnologia no cotidiano da enfermeira significa levá-la a compreender melhor o seu papel como administradora da assistência ao paciente no perioperatório, utilizando os novos recursos tecnológicos.

Entendendo que "as organizações utilizam alguma forma de tecnologia para executar suas operações e realizar suas tarefas"(6:2). Sabe-se que essa tecnologia poderá ser tosca e rudimentar ou poderá ser sofisticada, porém, todas as organizações dependem de um tipo de tecnologia para poderem funcionar e alcançar seus objetivos.

Observa-se que a introdução cada vez maior de elementos tecnológicos e científicos nos mais variados campos da ação humana, incluindo os serviços públicos e privados, exige a atualização de procedimentos de trabalho em velocidade que o ensino formal não consegue acompanhar.

Entende-se que na área de saúde, o crescimento tecnológico está acionado ao processo de trabalho e promove uma melhor qualidade de vida, reduzindo o sofrimento físico do paciente.

A lei do exercício profissional da enfermagem no Brasil, em seu artigo $8^{\circ}$, diz, na alínea h, que cabem ao enfermeiro, privativamente, "cuidados de enfermagem de maior complexidade técnica e que exijam conhecimentos adequados e capacidade de tomar decisões imediatas" e nas atividades como integrante da equipe de saúde, e na alínea $q$, "participação no desenvolvimento de tecnologia apropriada à assistência de saúde"(7:33).

Com base nessas constatações e reflexões, considerase que o conhecimento e domínio das novas tecnologias são de grande importância para a enfermeira de centro cirúrgico, que depende dela, para garantir um atendimento seguro ao paciente no perioperatório. Por meio dessas tecnologias, o paciente recebe o cuidado de que necessita; a enfermeira administra empregando diferentes equipamentos, técnicas e métodos.

Considera-se tecnologia biomédica os equipamentos utilizados em centro cirúrgico, que dão suporte à equipe de saúde nos cuidados prestados ao paciente no perioperatório; a tecnológicos da comunicação (fax, telefone, vídeo-conferência, entre outros), como forma de beneficiar seus pacientes e auxilialos na assistência, e, considera-se a tecnologia da informação como sistema de computadores que coletam dados, armazenam, processam, recuperam, mostram e comunicam a informação necessária, em tempo real( ${ }^{(8)}$.

Em relação a informação. ressaltam "o uso rotineiro de informações em um hospital atende às necessidades, a priori, de dois processos: a assistência ao paciente e o funcionamento do próprio hospital"(9:178).

Com o crescimento tecnológico, a informação, em especial, constitui o elemento básico para a prática da enfermagem perioperatória em centro cirúrgico, a fim de melhorar a qualidade da assistência que deve prestar ao paciente.

Então, percebe-se a necessidade de abordar as novas tecnologias biomédicas, da informação e comunicação em saúde, e em especial em enfermagem, que muito é e será utilizada pela enfermeira no gerenciamento de centro cirúrgico.

\section{Procedimentos Metodológicos}

Para alcançar os objetivos propostos, procurou-se desenvolver um estudo transversal exploratório-descritivo, por meio de uma abordagem quantitativa, a fim de possibilitar compreensão da maneira que a enfermeira de centro cirúrgico gerencia as novas tecnologias.

Este estudo foi autorizado pelo Comitê de Ética e Pesquisa da Universidade Estadual de Feira de Santana, o universo da presente investigação constitui-se de instituições hospitalares, cujo quadro de pessoal fosse composto por enfermeiras com experiência em unidade de centro cirúrgico e que estivesse em pleno exercício profissional no local da pesquisa.

Pesquisou-se 33 enfermeiras dos hospitais do Município de Feira de Santana-BA no período de junho e julho de 2002, a fim de que se pudesse entender melhor o gerenciamento das novas tecnologias da enfermeira em centro cirúrgico.

Nos hospitais, fez-se contato com os diretores, entregaram-se as solicitações para autorização da pesquisa, foram dadas as informações sobre os objetivos da pesquisa 
para estes, as chefias de enfermagem e as enfermeiras, informaram-se os objetivos da pesquisa e solicitou-se das mesmas a colaboração e autorização por meio do Termo de Consentimento Livre e Esclarecido; foi entregue, a cada enfermeira, o questionário e agendada a data de devolução.

O instrumento utilizado foi o questionário a fim de que fossem alcançados os objetivos traçados para o estudo.

\section{Processamento e tratamento estatístico}

Com base nas características (variáveis dependentes) levantadas em um questionário aplicado às enfermeiras dos centros cirúrgicos, criou-se uma nova variável dependente: nível de adequação ao domínio das novas tecnologias das enfermeiras no gerenciamento de centro cirúrgico em hospitais, com duas categorias (Satisfatória e Insatisfatória).

As duas categorias foram definidas ao considerar-se a nota média obtida de 12 questões que originou uma média geral das enfermeiras de 5,17 , que tratavam do uso das novas tecnologias pelas enfermeiras dos centros cirúrgicos. Assim, as enfermeiras daqueles centros cirúrgicas que atingiram a média maior ou igual a 3,14 (61\%) foram agrupadas na categoria Satisfatória, e as abaixo de 3,14 (61\%), na categoria Insatisfatória. Após a alocação das enfermeiras nessas duas categorias, podese associar o índice de adequação ao domínio das novas tecnologias, aos possíveis fatores ambientais, que são as características do hospital e fatores individuais, as características sociodemográficas e comportamentais das enfermeiras.

O teste estatístico adotado neste estudo foi o QuiQuadrado de Pearson, estabelecendo-se o nível de significância de $5 \%$. Quando o número de casos for menor que 20 ou o valor esperado abaixo de 5, uma alternativa foi aplicar-se o teste exato de Fischer. O nível de significância estabelecido para os testes foi de $5 \%$ ou 0,05 ( $p<0,05)$.

\section{Análise e discussão dos resultados}

Como mostra a tabela 1, o nível de adequação ao domínio de novas tecnologias foi insatisfatório, alcançando freqüência superior correspondente a $54,5 \%$ do total da população, foi satisfatório, com um percentual de $45,5 \%$.

Tabela 1 - Nível de adequação quanto ao domínio de novas tecnologias das enfermeiras com experiências de centro cirúrgico. Feira de Santana-BA, 2002.

\begin{tabular}{c|c|c}
\hline \multirow{2}{*}{ NÍVEL DE ADEQUAÇÃO } & \multicolumn{2}{|c}{ Freqüência } \\
\cline { 2 - 3 } & $\mathrm{N}$ & $\%$ \\
\hline Satisfatório & 15 & 45,5 \\
\hline Insatisfatório & 18 & 54,5 \\
\hline TOTAL & 33 & 100,0 \\
\hline
\end{tabular}

Fonte: Dados da Pesquisa.

Tabela 2 - Nível de adequação ao domínio de novas tecnologias associadas aos fatores sexo e idade das enfermeiras com experiências em centro cirúrgico. Feira de Santana - BA, 2002

\begin{tabular}{|c|c|c|c|c|c|c|c|}
\hline SÓCIO- DEMOGRÁFICO & \multicolumn{2}{|c|}{ Satisfatório } & \multicolumn{2}{|c|}{ Insatisfatório } & \multicolumn{2}{|c|}{ Total } & p- Valor \\
\hline SEXO & $\mathrm{N}$ & $\%$ & $\mathrm{~N}$ & $\%$ & $\mathrm{~N}$ & $\%$ & \\
\hline $\begin{array}{l}\text { Feminino } \\
\text { Masculino }\end{array}$ & 15 & $\begin{array}{c}45,5 \\
-\end{array}$ & $\begin{array}{c}18 \\
-\end{array}$ & $\begin{array}{c}54,5 \\
-\end{array}$ & 33 & 100 & - \\
\hline TOTAL & 15 & 45,5 & 18 & 54,5 & 33 & 100 & \\
\hline $\begin{array}{c}\text { IDADE } \\
20-30 \\
30-40 \\
40-50\end{array}$ & $\begin{array}{c}5 \\
10 \\
-\end{array}$ & $\begin{array}{c}15,2 \\
30,3 \\
-\end{array}$ & $\begin{array}{c}4 \\
10 \\
4\end{array}$ & $\begin{array}{l}12,1 \\
30,3 \\
12,1\end{array}$ & $\begin{array}{c}9 \\
20 \\
4\end{array}$ & $\begin{array}{l}27,3 \\
60,6 \\
12,1\end{array}$ & $* 0,6967$ \\
\hline TOTAL & 15 & 45,5 & 18 & 54,5 & 33 & 100,0 & \\
\hline
\end{tabular}

Fonte: Dados da pesquisa.

* Teste exato de Fisher

Observa-se, na tabela 2 acima que, o sexo feminino, na amostra estudada, encontra-se em sua totalidade (100\%), em $54,5 \%$ dessas enfermeiras o NADNT foi considerado insatisfatório, e em 45,5\%, satisfatório.

São vários os fatores que interferem na prática da enfermagem, entre eles, destaca-se: força de trabalho predominantemente feminina ${ }^{(10)}$.

Observou-se na tabela 3 , que para $42 \%$ das enfermeiras com jornada de trabalho de 6 horas, o domínio de tecnologias foi satisfatório e para 19,4\% não, enquanto que, para $29 \%$ das enfermeiras com jornada de 8 horas foi insatisfatório.

Observa-se que estatisticamente existe associação significante no nível de 5\%, ou seja, há evidência entre carga horária das enfermeiras ao domínio de novas tecnologias.

Infere-se que, a carga horária (8 horas), pode dificultar o desenvolvimento do trabalho da enfermeira, no que diz respeito a assistência ao paciente, principalmente, por ser o centro cirúrgico uma unidade estressante e com grande número de atividades complexas, além de possuir um número reduzido de funcionários para o serviço.
Tabela 3 - O nível de adequação ao domínio de novas tecnologias associado ao fator carga horária de trabalho das enfermeiras com experiência em centro cirúrgico. Feira de Santana-BA, 2000

\begin{tabular}{c|c|c|c|c|c|c}
\hline \multirow{2}{*}{$\begin{array}{c}\text { Carga } \\
\text { horária }\end{array}$} & \multicolumn{2}{|c|}{ Satisfatório } & \multicolumn{2}{c|}{ Insatisfatório } & \multicolumn{2}{c}{ Total } \\
\cline { 2 - 7 } & $\mathrm{N}$ & $\%$ & $\mathrm{~N}$ & $\%$ & $\mathrm{~N}$ & $\%$ \\
\hline 8 & 01 & 3,2 & 09 & 29,0 & 10 & 32,2 \\
\hline 6 & 13 & 42,0 & 06 & 19,4 & 19 & 61,4 \\
\hline outras & 01 & 3,2 & 01 & 3,2 & 02 & 6,4 \\
\hline Total & 15 & 48,4 & 16 & 51,6 & 31 & 100 \\
\hline
\end{tabular}

Fonte: Dados da pesquisa.

Teste exato de Fisher: $p$ - valor $=0,0059$

* Percentual obtido, excetuando-se duas (2) enfermeiras que não responderam a questão. 
Da da amostra estudada, observou-se que 90,9\% das enfermeiras atuam em hospitais gerais, destas $51,5 \%$ o domínio de novas tecnologias foi insatisfatório e $39,4 \%$, foi satisfatório.

Observa-se, entretanto que, estatisticamente, não houve significância em nível de 5\% de probabilidade entre domínio de novas tecnologias e tipo de hospital.

Em relação à capacidade do hospital percebe-se que, $36,4 \%$ das enfermeiras que trabalham em hospitais de médio porte, o domínio de tecnologias foi satisfatório e para $21,2 \%$ foi insatisfatório. Para os hospitais de grande porte, toda a amostra estudada, ou seja, para $24,2 \%$, o domínio de tecnologias foi insatisfatório. Observa-se que, estatisticamente, existe associação entre capacidade do hospital e o domínio de novas tecnologias no nível de $5 \%$ de probabilidade.

Neste sentido, existe um crescimento excessivo no quadro de pessoal dos hospitais de grande porte, com pouca desvantagem aos serviços voltados ao paciente ${ }^{(9)}$. Portanto, observa-se que existe um excesso de funcionários burocráticos e um déficit de profissionais qualificados, principalmente na área de enfermagem.

Tabela 4 - O nível de adequação domínio de novas tecnologias associado ao fator instituição. Feira de Santana-BA, 2000

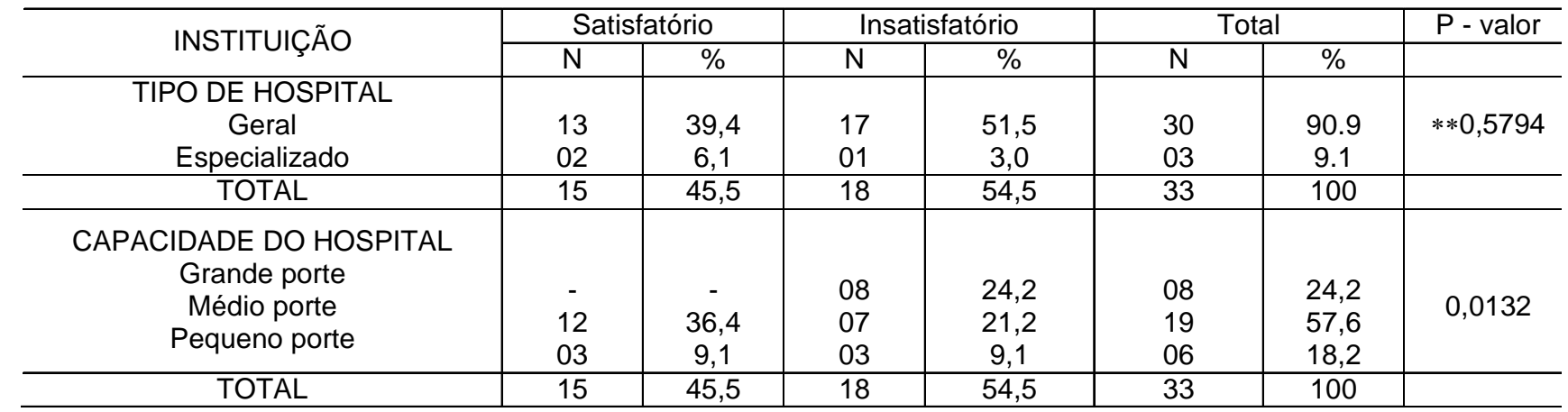

Fonte: Dados da pesquisa.

**Teste exato de Fisher

Tabela 5 - O nível de adequação ao domínio de novas tecnologias associado ao fator humanização das enfermeiras com experiência de centro cirúrgico. Feira de Santana-BA, 2002

\begin{tabular}{|c|c|c|c|c|c|c|c|}
\hline \multirow{2}{*}{ HUMANIZAÇÃO } & \multicolumn{2}{|c|}{ Satisfatório } & \multicolumn{2}{|c|}{ Insatisfatório } & \multicolumn{2}{|c|}{ Total } & \multirow[t]{2}{*}{$\mathrm{p}$-valor } \\
\hline & $\mathrm{N}$ & $\%$ & $\mathrm{~N}$ & $\%$ & $\mathrm{~N}$ & $\%$ & \\
\hline \multicolumn{8}{|l|}{$\begin{array}{l}\text { ORGANIZAÇÃO E } \\
\text { SISTEMATIZAÇÃO }\end{array}$} \\
\hline Sim & 11 & 34,4 & 16 & 50,0 & 27 & 84,4 & \multirow{3}{*}{0,6312} \\
\hline Não & - & - & - & & - & - & \\
\hline As vezes & 03 & 9,4 & 2 & 6,2 & 5 & 15,6 & \\
\hline *TOTAL & 14 & 43,8 & 18 & 56,3 & 32 & 100 & \\
\hline \multicolumn{8}{|l|}{ ASSISTÊNCIA DE } \\
\hline Sim & 8 & 25,0 & 9 & 28,1 & 17 & 53,1 & \multirow{3}{*}{0,6879} \\
\hline Não & 5 & 15,6 & 6 & 18,8 & 11 & 34,4 & \\
\hline As vezes & 1 & 3,1 & 3 & 9,4 & 04 & 12,5 & \\
\hline *2TOTAL & 14 & 43,7 & 18 & 56,3 & 32 & 100 & \\
\hline \multicolumn{8}{|l|}{ REDUZ A ASSISTÊNCIA } \\
\hline Sim & 1 & 3,1 & 4 & 12,5 & 5 & 15,6 & \multirow{3}{*}{$*^{4} 0,3547$} \\
\hline Não & 11 & 34,4 & 11 & 34,4 & 22 & 68,8 & \\
\hline As vezes & 2 & 6,3 & 3 & 9,4 & 5 & 15,6 & \\
\hline *3TOTAL & 14 & 43,8 & 18 & 56,3 & 32 & 100 & \\
\hline
\end{tabular}

Fonte: Dados da pesquisa.

*,*2,*3 Percentual obtido, excetuando-se uma enfermeira que não respondeu a questão.

${ }^{*}$ teste exato de Fisher

Na tabela 5 , observa-se que $84,4 \%$ das enfermeiras responderam que a tecnologia da informação contribui na organização e sistematização das atividades em centro cirúrgico.

Quanto à opinião que a tecnologia reduz, a assistência humanizada da enfermeira de centro cirúrgico, $68.8 \%$ das que responderam "não", 15,6\% enfermeiras que responderam "sim".

Essa associação demonstrou não haver significância no nível de 5\% de probabilidade entre o nível de adequação ao domínio de novas tecnologias com organização e sistematização, assistência de qualidade e reduz a assistência.

As inovações tecnológicas causam conflito tanto para o paciente quanto para a enfermeira; o paciente recebe o cuidado e a enfermeira o administra empregando diferentes equipamentos, técnicas e métodos. E acrescenta que o relacionamento humano é uma maneira de superar as conseqüências negativas da tecnologia, pois evita que os pacientes se transformem em meros objetos ${ }^{(10)}$.

A autora deste trabalho discorda que a enfermeira sente impacto com as inovações tecnológicas ${ }^{(11)}$. Sabe-se, que essa deve estar preparada para gerenciar as novas tecnologias, por meio de conhecimento científico e técnico, e carece usá-las na administração da assistência, garantindo um atendimento seguro ao paciente no perioperatório. 
Tabela 6 - Opinião das enfermeiras quanto ao gerenciamento de tecnologias em centro cirúrgico. Feira de Santana - BA, 2002.

\begin{tabular}{l|c|c|c|c|c|c|c|c}
\hline \multicolumn{1}{c|}{ VARIÁVEIS } & \multicolumn{2}{c|}{ SIM } & \multicolumn{2}{c|}{ NÃO } & \multicolumn{2}{c}{ AS VEZES } & \multicolumn{2}{c}{ TOTAL } \\
\cline { 2 - 8 } & $\mathrm{N}$ & $\%$ & $\mathrm{~N}$ & $\%$ & $\mathrm{~N}$ & $\%$ & $\mathrm{~N}$ & $\%$ \\
\hline Preparo para gerenciar & 25 & 75,8 & 08 & 24,2 & - & - & 33 & 100 \\
Investimento em equipamento & 32 & 97,0 & 01 & 3,0 & - & - & 33 & 100 \\
Participação na escolha e na aquisição** & 8 & 26,7 & 3 & 10 & 19 & 63,3 & 30 & 100 \\
Equipamento biomédico indispensável & 19 & 57,6 & 3 & 9,1 & 11 & 33,3 & 33 & 100 \\
Treinamento para o manuseio & 13 & 39,4 & 4 & 12,1 & 16 & 48,5 & 33 & 100 \\
Dificuldade no manuseio* & 5 & 15,6 & 9 & 28,1 & 18 & 53,3 & 32 & 100 \\
Controle diário* & 26 & 81,3 & 1 & 3,1 & 5 & 15,6 & 32 & 100 \\
\hline
\end{tabular}

Fonte: Dados da Pesquisa.

* uma enfermeira não respondeu a questão.

** três enfermeiras não responderam a questão.

Verificou-se na tabela 6 , que $75,8 \%$ das enfermeiras opinaram que estão preparadas para gerenciar as novas tecnologias em centro cirúrgico, entretanto, em 54,5\%, o domínio das novas tecnologias em centro cirúrgico foi insatisfatório. Fica claro que as enfermeiras com experiência em centro cirúrgico concordam que a tecnologia é importante no seu trabalho, promove um atendimento seguro, porém, não sentem segurança para usá-la na contribuição da assistência ao paciente. Acreditase que a enfermeira deve preparar-se melhor para gerenciar as tecnologias, o que com certeza pode-se conseguir por meio da atualização contínua.

A eficiência e a qualidade do suporte agregado pelo investimento em tecnologia dependem do preparo de seus usuários. Profissionais de todas áreas são desafiados o tempo todo. Para isso, é necessário estímulo e força de vontade para desbravar novos horizontes sem medo do desconhecido(13). Novas oportunidades estão emergindo com toda força, principalmente para o setor de enfermagem, e o único jeito de se precaver contra essa constante mudança é conhece-la, identificá-la e saber caminhar com ela.

Os atuais recursos científicos e tecnológicos têm possibilitado grandes avanços na realização de ações no atendimento à saúde da população. Enquanto que o uso de métodos manuais mostra-se cada vez mais ineficientes ${ }^{(7)}$.

Com relação à participação das enfermeiras na escolha e aquisição de materiais de consumo e permanente nas instituições que trabalham, $63.3 \%$ informaram que às vezes elas participam na escolha e $26.7 \%$ responderam que sim.

Infere-se que na área de equipamentos e materiais é da responsabilidade da enfermeira, portanto uma assistência de qualidade aos pacientes no perioperatório comumente depende da qualidade dos materiais e dos equipamentos. Assim, as enfermeiras deveriam estar familiarizadas com os materiais que usam, principalmente por ser da sua competência o planejamento e escolha dos mesmos.

Procurou-se saber das enfermeiras a importância dos equipamentos biomédicos na assistência de qualidade em centro cirúrgico, $57.6 \%$ responderam que acham indispensáveis esses equipamentos. Observa-se que as enfermeiras concordam com a importância da aquisição de equipamentos de alta tecnologia, ademais, percebe-se a dificuldade em utilizálos como contribuição na assistência.

Considerando a importância da atualização e do conhecimento da enfermeira, com referência ao manuseio dos novos equipamentos no centro cirúrgico, constatou-se que $59.4 \%$ responderam que "as vezes" sentem dificuldades em manusear os equipamentos no centro cirúrgico.

Acredito que os profissionais de saúde estão seguros da importância que as tecnologias trazem, porém, ressalto que a operacionalização ainda deixa a desejar ${ }^{(12)}$.
Assim, entende-se que a maior dificuldade das enfermeiras no manuseio dos equipamentos e materiais devese aos seguintes fatores: sua ausência na participação e escolha desses materiais durante o planejamento da unidade; despreparo em decorrência da falta de treinamento e atualização e a falta de oportunidade em utilizá-los devido ao excesso de atividades administrativas e burocráticas.

Tabela 7 - A enfermeira e a informatização em centro cirúrgico. Feira de Santana - BA, 2002

\begin{tabular}{c|c|c}
\hline Variáveis & $\mathrm{N}$ & $\%$ \\
\hline IMPORTÂNCIA DA & & \\
INFORMATIZAÇÃO & 32 & 97 \\
Sim & - & - \\
Não & 1 & 3 \\
As vezes & 33 & 100 \\
\hline TOTAL & & \\
\hline CENTRO CIRÚRGICO & 14 & 43.8 \\
INFORMATIZADO* & 18 & 56.3 \\
Sim & 32 & 100 \\
Não & &
\end{tabular}

Fonte: Dados da pesquisa.

Constatou-se na tabela 7 , que $97 \%$ das enfermeiras consideram importante a informatização do centro cirúrgico.

Apesar de a tecnologia da informação ter avançado rapidamente no item referente à conectividade física para comunicação da informação, aspectos referentes à integração de sistemas e utilização da informação pelos profissionais de saúde não avançaram no mesmo nível(13).

As profissionais de enfermagem, pelo que se observa, não dominam a tecnologia da informação, principalmente por carência de enfermeiras especializadas nessa área, pouca motivação em aprender a lidar com a informática, e a falta de preocupação de alguns hospitais em acompanhar o atual desenvolvimento tecnológico.

Inquiridos com relação às enfermeiras que trabalham em hospitais que têm o centro cirúrgico informatizado, verificouse que $56,3 \%$ (tabela 7 ) das enfermeiras trabalham em hospitais em que o centro cirúrgico não é informatizado.

Neste sentido, a informatização, o trabalho da enfermeira será mais organizado e eficiente, eliminando atividades redundantes e ineficazes, podendo medir resultados de suas ações de forma mais direta, adverte, entretanto, que qualquer desenvolvimento de sistemas informatizados está na dependência de quem o desenvolve ${ }^{(14)}$. 
Portanto, diante de um percentual maior para os centros cirúrgicos não informatizados, percebe-se pouca intimidade da enfermeira com a tecnologia da informação, no que concerne a sua utilização no apoio à prática da enfermagem.

\section{Conclusão}

Conclui-se que há evidência de que as enfermeiras necessitam gerenciar o centro cirúrgico por meio de atividades sistematizadas e com adequação ao nível satisfatório do domínio de novas tecnologias (biomédicas, comunicação e informação).

O nível de adequação da enfermeira ao domínio de novas tecnologias (NADNT) no gerenciamento em centro cirúrgico foi insatisfatório $(54,5 \%)$, não se pode afirmar existir só o exercício desse padrão, estando a gerência interligada a outras situações que são aplicados na prática da enfermeira na referida unidade.

Comprovou-se estatisticamente haver discordância significativa em nível de $5 \%$ de probabilidade entre as variáveis categorizadas no perfil da enfermeira e característica da instituição com o NADNT, exceto as seguintes variáveis: carga horária e capacidade do hospital. Entretanto, observa-se que as demais variáveis (sexo, idade, tipo de hospital) são fatores condicionantes no domínio das novas tecnologias. Pelo exame percentual e a vivência profissional da autora, constata-se a importância que cada uma das varáveis representa para o NADNT pela enfermeira de centro cirúrgico, pois refletem eficácia, qualificação do individuo, conhecimento, habilidades (técnica, humana, liderança, tomada de decisão), aspectos referenciados pela literatura, com fatores condicionantes do êxito do gerenciamento de novas tecnologias em centro cirúrgico.

Considerando os resultados alcançados, adverte-se que a realidade da prática representa a tendência do grupo a uma conscientização do seu verdadeiro papel de gerente de centro cirúrgico frente às novas tecnologias.

\section{Referências}

1. Tramontini CC, Lopes DFM, Kikuchi EM, Kemmer LF, Garanhani L. Repensando a formação do gerente do processo de trabalho do enfermeiro de centro cirúrgico e central de material. Revista SOBECC, São Paulo 2002 jan/mar;7(1):11-5.

2. AORN. Categoria dos padrões administrativos para enfermagem perioperatória - parte I. Revista SOBECC, São Paulo 2002 abr/jun; 7(2):8-10.

3. Servo MLS. Supervisão em enfermagem: o (re)velado de um práxis. Feira de Santana (BA): Universidade Estadual de Feira de Santana; 2001.

4. Cunha AMCA. Gestão em enfermagem: novos rumos. Revista O Mundo da Saúde, São Paulo 2002 abr/jun;26(2):309-14.

5. Grinspunm MPSZ, Rodrigues AMM, Neves AMC, Cardoso TFL, organizadores. Educação tecnológica: desafios e perspectivas. São Paulo: Cortez; 1999.

6. Chiavenato I. Introdução à teoria geral da administração. São Paulo: Makron Book; 1997.

7. Lei n. 7.498 , de 25 de junho de 1986, que dispõe sobre o exercício da enfermagem, e dá outras providências. COREN-BA: Salvador; 1999.

8. Marin HF. Tecnologia da Informação em enfermagem: próximos passos. Revista O Mundo da Saúde, São Paulo 2000 maio/jun;24(3):200-4.

9. Ferreira DP, Lira ACO. O papel da informação no hospital do futuro. Revista O Mundo da Saúde, São Paulo 2000 mai/jun;24(3):173-81.

10. Capella BB, Gelbcke FL. Enfermagem: sua prática e organização. Revista Brasileira Enfermagem, Brasília (DF) 1988 abr/jun;42(2);13239.

11. Groah LK. Enfermagem perioperatória: a essência de qualidade e do cuidado. Revista SOBECC, São Paulo 1997 jul/set;2(3):12-7.

12. Muhlen SS. O engenheiro clínico nas unidades de saúde. Revista Hospital: Unimed do Brasil, São Paulo 1995 nov;26-7.

13. Amaral MB. Tecnologia da informação e gestão em saúde. Revista $O$ Mundo da Saúde São Paulo 2002 abr/jun;26(2):225-32.

14. Marin HF. Informática à serviço da enfermagem. Revista Nursing, São Paulo 1999 mar; 2(10): 8-9.

Data de Recebimento: 20/02/2004

Data de Aprovação: 24/08/2004 